\title{
DESIGN OF A VEHICULAR TRACKING SYSTEM USING COMBINED CED ALGORITHM AND FUZZY LOGIC
}

\author{
Vidyashree.K ${ }^{1}$, K.V.Mahendra Prashanth ${ }^{2}$ \\ ${ }^{1}$ Post Graduate Student, Department of VLSI and Embedded Systems, SJBIT College of Engineering, Karnataka, India \\ ${ }^{2}$ Professor, Chief Co-coordinator, R\&D and PG Programmes, Department of E\&CE, SJBIT College, Karnataka, \\ India
}

\begin{abstract}
Edge detection is the most popular and commonly used technique for an image analysis. Much evaluation, experiments and deployment is done on edge detection. The major problem in edge detection technique is discontinuity in the image brightness in the different regions of an image. In order to avoid this particular issue, combination of two edge detection algorithms have been proposed, canny edge detection algorithm and Fuzzy based edge detection algorithm. In proposed method, along with tracking, classification of vehicles is also determined by using the distance Euclidean square algorithm and Kalman filter. The position of each vehicle will be estimated and tracked. The Kalman filter classifies detected vehicles in different specified groups and count them separately to provide useful information for traffic flow analysis. On observing the obtained results it is concluded that fuzzy canny edge detection has better efficiency than canny algorithm alone. This approach has provided improved results over the traditional canny edge detection technique based on Gaussian filter for noisy images.
\end{abstract}

Keywords: Canny Edge Detection Algorithm, Fuzzy Algorithm, Kalman Filter and Image Edge Detection.

\section{INTRODUCTION}

In the field of image processing some of the significant areas to be considered are image edge detection, locating and identifying the discontinuities and boundaries between many areas in an image, which defines the image edge detection process. Edge detection is the most popular and commonly used for an image analysis. Earlier traditional edge detection techniques were depending on linear methods and there will be more discontinuity in the images; due to its variable brightness, however by using proposed system and algorithms non-linear methodology can be deployed to reduce discontinuity in images which was present in the conventional methods of image edge detection.[1] Z. Zhang et.al describes an end-to-end method for extracting data of moving targets from an input video, according to image based properties targets are classified into different classes, and then strongly tracking them. Moving targets are detected using the difference of pixels between consecutive image frames. The resulting system strongly identifies region of interest, rejects background clutter; and continually tracks over large distances and periods of time despite occlusions, appearance changes and completion of target motion. V. Mittal et.at [2] proposed canny edge detection and Gaussian filter to remove noise in an image. This method categorizes the boundary of an object and reduces the problem of optimization problem. Ohnishi, $\mathrm{N}$ et.al [3] proposes the FEDGE - Fuzzy edge recognition by fuzzy categorization and classification of edges. In this processed images are categorized into different classes based on fuzzy logic. A system based on robust vehicles extraction in a video-based intelligent transportation system for tracking the vehicles in traffic is reported in paper [4]. Marr, D et.al [5] proposed a system; based on the image edge detection using fuzzy logic and end-to-end method for extracting information of moving targets from an input video. According to image based properties targets are categorized and tracked based on different classes [6].Controlling traffic at the intersection has been demonstrated and tested along with a real-time computer simulation. Further OpenCV tool is used for the dissection of images of empty lanes and the information obtained from them is stored. An improved Canny Edge Detection Algorithm using morphological filters; which helps to identify prominent object with less number of edges [7].In this approach edge detected is less compared to simple Canny edge detection. Classification of passing vehicles in roadways using an image processing techniques and Kalman filter algorithm is proposed by Ali Tourani et.al [8].In reference [9], fuzzy rule based edge detection algorithm were used to determine the vehicle density on a particular lane and control the signal accordingly. Further fuzzy edge detection method proved to be more accurate and efficient as compared to standard edge detection methods. Kavya. P.Walad et.al [10] proposed efficient fuzzy logic based algorithm was employed to detect vehicle edges in an input image by scanning it through the $2 * 2$ mask. Triangular membership functions of Mamdani type fuzzy inference system is used for inputs containing fuzzy sets and output containing fuzzy set. Based on these sets of rules the output of fuzzy is preferred that particular pixel is edge or not. Sobel edge detection technique and Prewitt edge detection technique were being implemented for detection of edge but these two techniques suffer from problems: edge dislocation, disappearing edges and false edges. Many traditional techniques for image edge detection failed to improve the image quality. Canny edge detection technique proves to be more accurate than other edge detection 
techniques but it shows inadequate results on low contrast images and noisy images. To avoid this issue, in this paper combination of two edge detection algorithms are proposed; canny edge detection algorithm and fuzzy based edge detection algorithm. In proposed system almost all the edges in an image are identified on the basis of fundamental elements and the color, quality and gray scale features.

Canny edge detection is used along with the Kalman filter for tracking the vehicles; which is nonlinear method which enables tracking the object by object detection methods. This paper focuses on employment of an efficient, simple, cost effective method of tracking vehicles in highway with less human involvement. The objective of the present work was to track vehicles in the highway from dynamic data obtained from the camera using combined canny edge detection algorithm and fuzzy logic edge detection technique.

\section{IMPLEMENTATION}

The block diagram of the implementation schematic of the proposed system is shown in figure 1; in which tracking movement of vehicles is determined in the given input video. The given input video is subjected to generate the frames for the particular video and after generation of frames pre-processing of input image frames is applied, where RGB images is converted into grey scale images. The converted gray scale images are subjected to image edge detection techniques. Canny image edge detection algorithm and also fuzzy image edge algorithm are used for detecting the boundaries of an image. An hybrid approach is implemented; where first phase of edge detection implements fuzzy rule based system and second phase of edge detection implements Canny edge detection to obtain satisfactory results. The motion analysis of vehicles in the input video is measured by optimal floor using Lucaskande algorithm which defines the motion of vehicle. Next by applying Kalman filter, the object tracking process is done, which helps for object detection and tracking in a considering gray scale image. On the basis of the result obtained from the object tracking, Kalman's filter assists to classify the vehicles using Euclidean distance square algorithm.

\subsection{RGB to Gray Scale Conversion}

In order to reduce the cost of computation while not having to compromise on the proficiency, after the generation of the frames extraction RGB to Gray scale conversion is done. The lookup table of a RGB image has 24 bits ( 8 bits each for $\mathrm{R}, \mathrm{G}$ and $\mathrm{B}$ since each may have values ranging from $0-255$ ) whereas lookup table of gray scale image has only 8 bits (values from 0-255). It has been observed that edge detection algorithms produce relative results for both RGB and gray scale images.

\subsection{Edge Detection Using Fuzzy Logic}

The fuzzy technique is an operator introduced in order to simulate at anexact level the compensatory behavior in process of decision making. It appears that fuzzy methodologies deliver more operative results than any other existing systems. Image gradient in fuzzy logicis the method which is utilized to extract data from images. The implication of membership functions is a delicate point in the framework of fuzzy controller. In proposed system triangular membership function is used due to its simplicity which requires only three parameters to define. The sample triangular membership function is given as follows respectively. The triangular curve is functions of a vector, $\mathrm{X}$, and depends on three scalar parameters A, B, and C

$f(X ; A, B, C)=\left\{\begin{array}{cl}0 & , X \leq A \\ \frac{X-A}{B-A}, & A \leq X \leq B \\ \frac{C-X}{C-B}, & , B \leq X \leq C \\ 0 & , C \leq X\end{array}\right.$

The parameters ' $\mathrm{A}$ ' and ' $\mathrm{C}$ 'locate the "base" of the triangle and the parameter 'B'locates the peak. In proposed system input to the fuzzy inference (FIS) system are obtained by applying a high-pass filter to original image, a first-order edge detector filter (Sobel operator) and a low-pass (mean) filter.

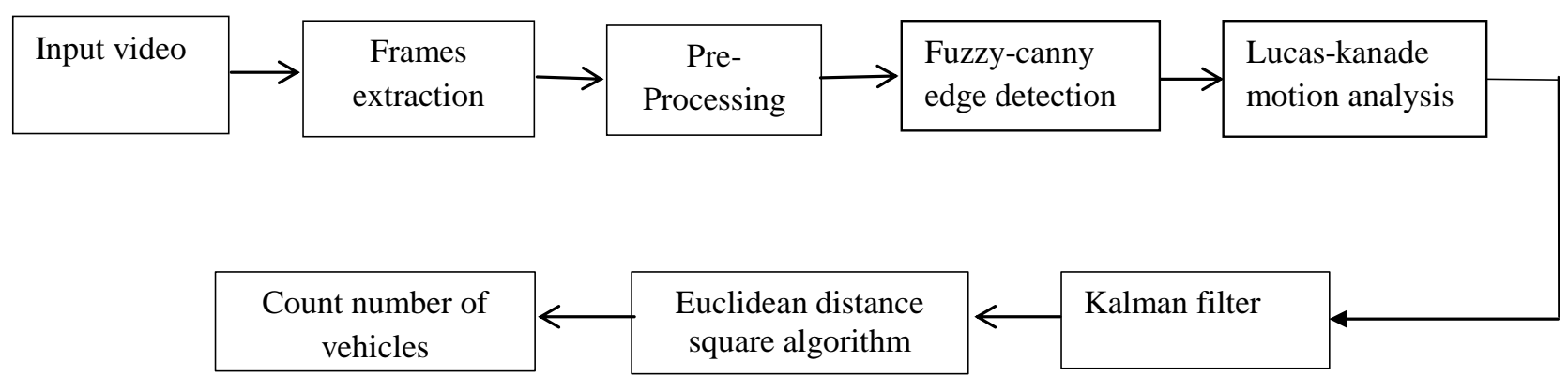

Figure 1:Block Diagram of implementation schematic of the proposed system

\subsection{Canny Edge Detection Algorithm}

Canny edge detector is widely considered to be the standard edge identification algorithm. There are several steps to be followed in the canny edge detector, firstly smoothen of the image is done with a two dimension Gaussian filter, image gradient of a considered input image is considered, suppression is done through non-maximal and then threshold of each edge is determined. The goal of this edge detection algorithm is to remove moving edges from 
continuous video to get quantifiable geometric estimations of passing vehicles. Using the gradient kernel approach image gradient magnitude is determined in both horizontal direction Gx and vertical direction Gy for each pixel. And the direction of the pixel is measured by

$\theta=\arctan (\mathrm{Gy} / \mathrm{Gx})$

Limit values such at the higher threshold value (HTV) and lower threshold value (LTV) are chosen from the histogram of the image so as to recognize the edges.

1. The gradient of the pixel is more protruding than the HTV the logic that pixel is taken as edge pixel.

2. If the gradient lie between high and lower threshold value in the logic, that value is also consider as edge pixel.

3. If the gradient value is lower than the LTV in the logic that is declared as non-edge pixel.

This lower and higher threshold values are checked by the observation of the examinations. These two threshold values are used to identify large edges and to find the small edges.

\subsection{Fuzzy-Canny}

In this proposed system, the hybrid approach is proposed and implemented. This approach suggests two phases for edge detection. First phase deals with fuzzy rule based system and second concerns about canny edge detection. This proposed has been implemented and the better results have been obtained for edge detection. Fuzzy system is used in canny edge detection to overcome the weakness of low contrast image. Due to intensity variability canny edge detection technique gives less quality of image. For further more improvement in the quality of image, Fuzzy logic is applied to the canny edge detected image. The flowchart of proposed methodology for fuzzy-canny is shown in Fig2.

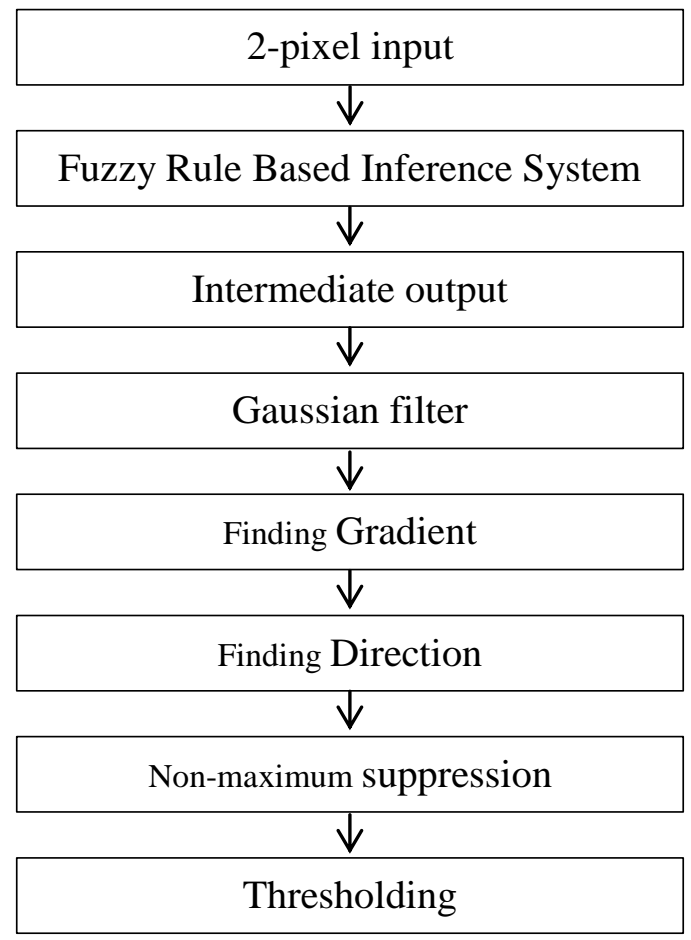

Figure 2: Flowchart of fuzzy-canny edge detection

\section{Phase 1 of Edge Detection}

\section{Input: RGB to gray-scale converted image}

Step 1: Obtain the gradient along magnitude and direction for image gradient using Sobel operator as an input, output of the Sobel is obtained before applying fuzzy rules.

Step 2:Triangular Membership function is applied for the fuzzy inference system where pixels are taken as a crisp input for FIS system, to be converted into morphological variable i.e. black and white, by using triangular membership function.

Step 3: If else rules is applied as fuzzy rules to obtain output image as given

If

Fx is zero and Fy is zero then Fout is white

Else If

Fx is not zero or Fy is not zero then Fout is black

where Fx and Fy are input of image gradient along $\mathrm{x}$-axis and $y$-axis and Fout is the desired Output.

Step 4: Fuzzy output is defuzzified using centroid method to get the intermediate output.

The Fuzzy output retrieved from the Fuzzy Inference System is considered as an input to canny edge detection technique.

\section{Phase 2 of edge detection}

\section{Input: Intermediate Image from phase 1.}

\section{Output: Resultant fuzzy canny output image with edge detection.}

Step 1:Gaussian filter is applied for flattening the image

Step 2: Gradient and direction of transitional output image is determined.

Step 3: By applying non-maximum suppression, weak edges are suppressed to get thin line.

Step 4: Edge threshold is determined, and then final output image is obtained.

\subsection{Lucas-Kanade Algorithm}

After final output edge detection image is found then motion analysis of vehicles in the input video is measured by optimal floor using Lucas-Kanade algorithm which defines the motion of vehicle. If the increase in brightness per pixel at $(\mathrm{x}, \mathrm{y})$ is $\operatorname{Px}(\mathrm{x}, \mathrm{y})$ is the $\mathrm{x}$-direction, and the increase in brightness per pixel in the $y$ direction is $\operatorname{Py}(x, y)$, then there will be a total increase in brightness, after a movement by $u$ pixels in the $\mathrm{x}$ direction and $\mathrm{v}$ pixels in the $\mathrm{y}$ direction at movement vector $\mathrm{u}$ and $\mathrm{v}$ : 
$P_{x}(x, y) \cdot u+P_{y}(x, y) \cdot v$

$P_{x}(x, y) \cdot u+P_{y}(x, y)=-P_{t}(x, y)$

Based on proposed methodology of background subtraction, vehicles are detected in three sequential frames. When a moving vehicle is detected, a bound whelming vehicle borders in binary image is drawn which is further used for counting and classification of vehicles.

\subsection{Kalman Filter}

Kalman filtering technique is used for vehicle tracking and classify passing vehicles in different categories. In roadway videos, the edge detection function provides an imprecise position of vehicles movement, but the knowledge of the vehicle's current position needs to be improved. The Kalman filter can optimally estimate the position of each vehicle and predict the location of vehicles in video frames by minimizing noise disorders.

\subsection{Counting and Classification Function}

Classification of the data is based on the training set and features obtained using Gray Level Co-occurrence Matrixalgorithm. Euclidean Distance square algorithm is used to assign incoming vehicle feature vectors into different categories. Class of query vehicle feature vector is determined based on lowest distance value obtained between template vector and feature vector. In Euclidean distance square method, distance is measured between two vectors let us consider $\mathrm{u}$ and $\mathrm{v}$ are two vectors then Euclidean distance is given as $d(u, v)=\sqrt{\sum_{i}^{n}\left(u_{i}-v_{i}\right)^{2}}$

By using equation (5) the distance between the vehicles is calculated by considering the minimum value of the result which is further used for classification of the vehicles according to the categories saved in database during training phase.

The proposed system is developed to classify vehicles into three different classes based on their size and shape features extracted from vehicle side view. In this work, vehicles are grouped into three classes, they are: Class 1(bicycles, motorcycles), Class2 (Motorcars) and Class3 (Bus, lorry, trailers)

A counter is used to count only the vehicles passing over the detection zone. In this technique counting is according to the number of moving vehicles detected in the detection zone and classified in one of the mentioned groups.

\subsection{Comparative Analysis}

This paper provides the comparison results for canny edge, fuzzy edge and fuzzy-canny edge. True rate has improved in detecting the number of vehicles correctly and has reduced the false rate where the vehicles detection occurs even in their absence due to noise in the images with less than $10 \%$ as shown in figure 3 .

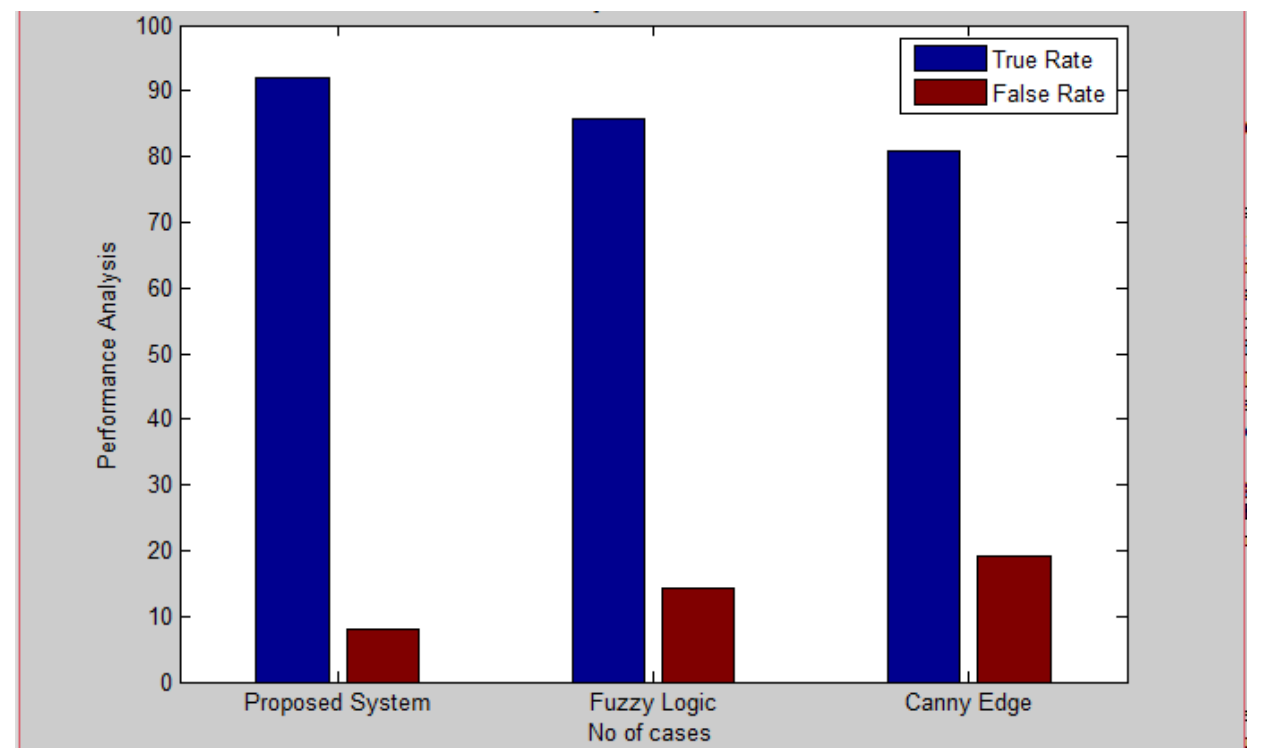

Figure 3: Comparative analysis

\section{EXPERIMENTAL RESULTS}

The experimental result for the above discussed methodology is discussed in this section. The proposed combination approach of fuzzy-canny is implemented in Matlab and gives improved results than classical canny method.
Figure 3(a) to figure 3(h) show the results obtained by proposed approach. Figure 3(a) shows the original images. Figure 3(b) shows the RGB to gray scale converted images. Figure 3(c) shows the result of Fuzzy edge detection. Figure 3(d) provides the output of Canny edge detection. Figure 3(e) shows the resultant image after application of hybrid approach. 
As observed, the hybrid approach provides accurate and more improved results than figure 3(c) and figure 3(d).

A motion detected image is also considered for further processing in order to detect the objects using Lucas-kanade algorithm which estimate the movement of interesting
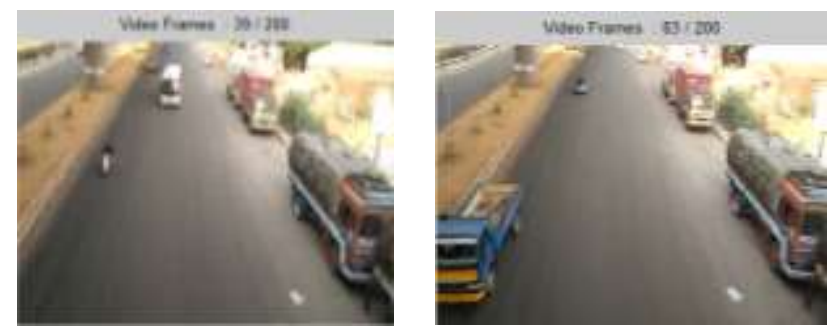

Figure 3(a)

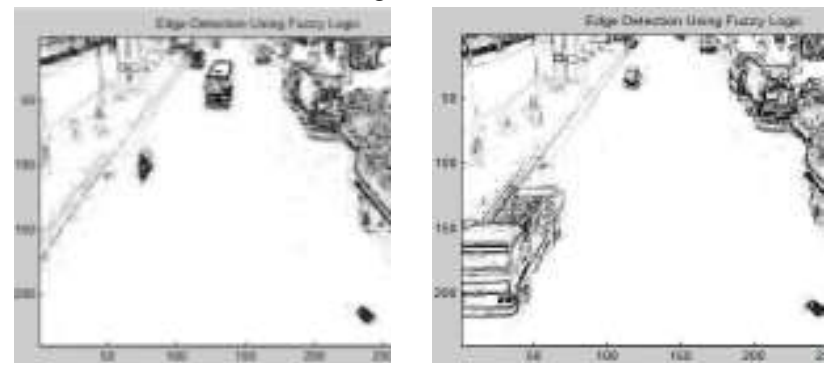

Figure 3(c)
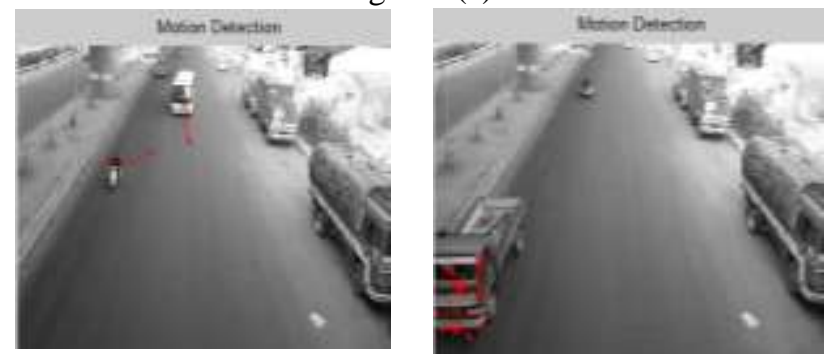

Figure 3(f)

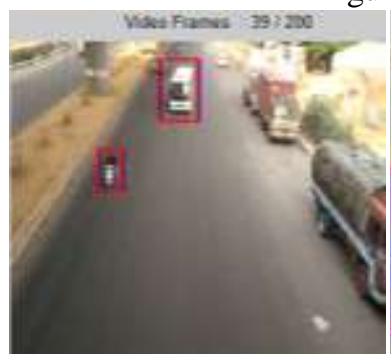

Figure 3(h)

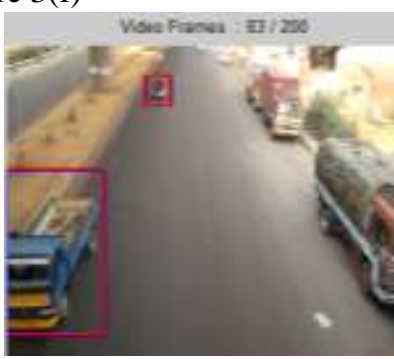

Figure 3(i) features in successive images. The results are shown in Figure 3(f) and 3(g). The estimation of current position of each vehicle is obtained using Kalman filter shown in figure 3(i). Fig. 3(j)gives number of vehicles counted and classified using distance Euclidean square algorithm for each frame.
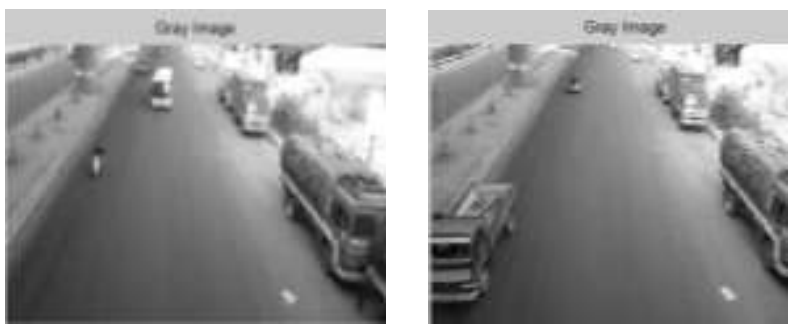

Figure 3(b)
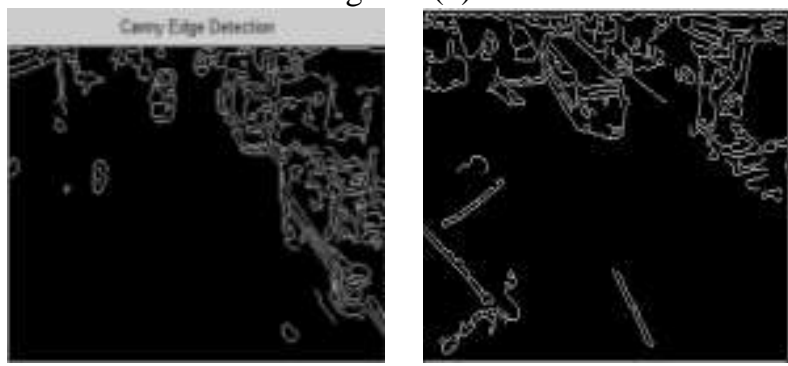

Figure $3(d)$

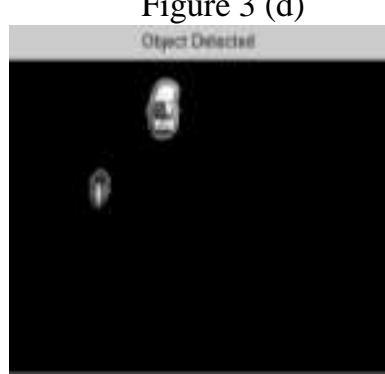

Figure 3 (e)

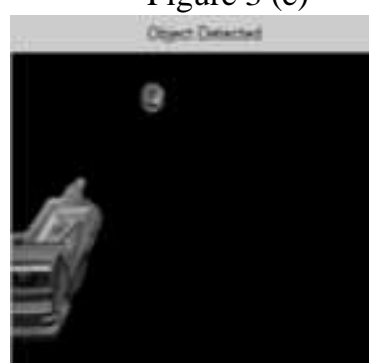

Figure 3(g)

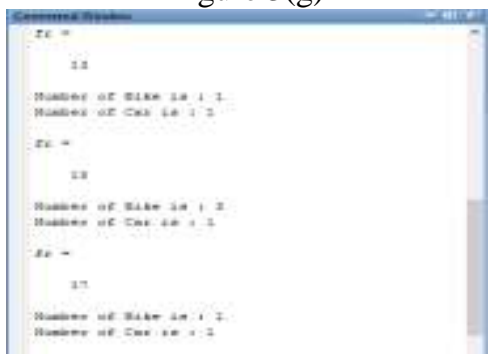

Figure 3(j)

Figure 3: (a)Input image; (b) Gray scale image; (c) Fuzzy logic ;(d) Canny detection image;(e)fuzzy-canny edge detection image (f) Motion detection image;(g) object detected image; (h) and (i) Tracked images;(j)snapshot of number of vehicles counted and classified for each frame

\section{CONCLUSION}

In this paper, fuzzy canny technique were applied for traffic video which gives better results when compared to other edge detection techniques for vehicular tracking system. This technique provides improved results in detecting the number of vehicles and has reduced the false rate where the vehicles detection occurs even in their absence due to noise in the images. This technique has a reduced false rate of less than $10 \%$ as compared to other edge detection techniques. On observing the obtained results it is concluded that fuzzy canny edge detection has better efficiency than canny algorithm alone. This approach has provided improved results over traditional canny edge detection technique based on Gaussian filter for noisy images.

Further work needs to be carried out to reduce number of false ratewhile detecting vehicles which are very close together by adapting efficient morphological operations along with edge detection techniques. 


\section{REFERENCES}

[1].Z. Zhang, Y. Cai, K. Huang, and T. Tan, "Real-time moving object classification with automatic scene division," in Proc. IEEE Int. Conf. Image Process., San Antonio, TX, 2007, vol. 5, pp. V-149-V- 152 V.

[2].Mittal and M. S. Batra, "Edge Detection Technique by Using Nero Fuzzy System,", RIMT-IET, Mandi Gobindgarh. March 29, 2008.

[3].Ho, K.H.L., and Ohnishi, N., "FEDGE - Fuzzy edge detection by fuzzy categorization and classification of edges", Fuzzy Logic in Artificial Intelligence, Springer, IJCAI'95 Workshop, Montreal, Canada, 1188:182- 196.

[4].L. Xie, G. Zhu, Y. Wang, H. Xu, and Z. Zhang, "Robust vehicles extraction in a video-based intelligent transportation system," in Proc. IEEE Int. Conf. Commun., Circuits Syst., Hong Kong, China, 2005, vol. 2,pp. 887-890. [5].Marr, D., and Hildreth, E.C., "Theory of edge detection",Proc. of the Royal Society of London, b207, 1980, 187-217.

[6].Sachin Grover, Vinay Shankar Saxena, Tarun Vatwani, "Design of Intelligent traffic control system using Image Segmentation" International Journal of Advances in Engineering \& Technology, Nov., 2014.

[7].Weibin Rong, Zhanjing Li, Wei Zhang and Lining Sun, "An Improved Canny Edge Detection Algorithm", Proceedings of 2014 IEEE International Conference on Mechatronics and Automation, Tianjin, China August 3 - 6, 2014.

[8].Ali Tourani "Vehicle Counting Method Based on Digital Image Processing Algorithms" 978-1-4799-84459/15/\$31.00 2015 IEEE

[9].Sreemana Datta,Joydeep Mukherjee," Real-Time Traffic Control System using Fuzzy Logic based Edge Detector for Images" International Journal of Computer Applications (0975 - 8887)Volume 119 - No.14, June 2015

[10].Kavya P Walad,Jyothi Shetty ,"Fuzzy Logic Based Vehicle Edge Detection Using Trapezoidal and Triangular Member Function" International Journal of Engineering Research and General Science Volume 3, Issue 1, JanuaryFebruary, 2015

\section{BIOGRAPHIES}

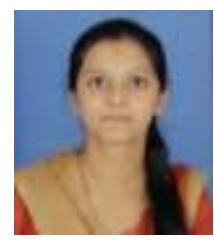

Vidyashree.K obtained B.E degree in Electronics and Communication Engineering at A.C.S college of engineering, Bangalore and currently pursuing M.Tech degree in VLSI Design and Embedded Systems from Visvesvaraya Technology University, Belgavi. My areas of interests are image processing, VLSI design and embedded systems.

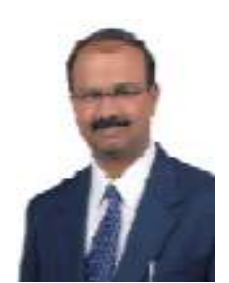

Dr. K V Mahendra Prashanth B.E., M.Tech, Ph.D.

Dr. K.V. Mahendra Prashanth has obtained his B.E. degree in Electronics \& Communication Engineering at National Institute of Engineering, Mysore; M.E. degree in Power Electronics from UVCE Bangalore. He received his Ph.D. degree in Electronics \&
Communication Engineering from Visvesvaraya Technological University, Belagavi. His area of interests are signal processing, active noise control, Bio-Medical engineering. He has around 23 years of teaching experience. Presently, he is working as Professor in Department of Electronics \& Communication Engineering, Chief Coordinator for R\&D and PG programmes at SJBIT, Bangalore. He has published several papers in international and National journals, and presented papers in International conferences. He is a Reviewer for international \& National Journals. He is the Member of IEEE, Acoustical society of India, ACCS, IIAV, ISTE etc. 